\title{
Alleviating Eavesdropping Attacks in Software-Defined Networking Data Plane
}

\author{
Ahmad Aseeri \\ Department of Computer Science \\ Whitacre College of Engineering \\ Texas Tech University \\ Lubbock, Texas 79409 \\ ahmad.aseeri@ttu.edu
}

\author{
Nuttapong Netjinda \\ Computer Engineering Department \\ King Mongkut's University of \\ Technology Thonburi \\ Bangkok, Thailand \\ nuttapong.net@gmail.com
}

\author{
Rattikorn Hewett \\ Department of Computer Science \\ Whitacre College of Engineering \\ Texas Tech University \\ Lubbock, Texas 79409 \\ rattikorn.hewett@ttu.edu
}

\begin{abstract}
Software-Defined Networking (SDN) is an emerging paradigm that introduces a concept of programmable networks to enhance the agility in networking management. By separating concerns of the data plane and the control plane, implementing network switching as packet forwarding, and using centralized software to logically control the entire networks, SDN makes it simpler to automate and configure the network to respond to high-level policy enforcement and dynamically changing network conditions. As SDN becomes more prevalent, its security issues are increasingly critical. Eavesdropping attacks are one of the most common and important network attacks because they are relatively easy to implement and their effects can escalate to more severe attacks. This paper addresses the issue of how to cope with eavesdropping attacks in the SDN data plane by using multiple routing paths to reduce the severity of data leakage. While this existing approach appears to be considerably effective, our simple analysis uncovers that without a proper strategy of data communication, it can still lead to $100 \%$ of data exposure. The paper describes a remedy along with illustrations both analytically and experimentally. The results show that our proposed remedy can avoid such catastrophe and further reduces the percentage of risk from data exposure approximately by a factor of $\frac{1}{n}$ where $n$ is the number of alternate disjoint paths.
\end{abstract}

\section{CCS CONCEPTS}

- Security and privacy $\rightarrow$ Network security;

\section{KEYWORDS}

SDN, OpenFlow, Anti-Eavesdropping, Multipath Routing.

\section{ACM Reference format:}

Ahmad Aseeri, Nuttapong Netjinda, and Rattikorn Hewett. 2017. Alleviating Eavesdropping Attacks in Software-Defined Networking Data Plane. In Proceedings of 12th Annual Cyber and Information Security Research Conference, Oak Ridge, TN, USA, April 04 - 06, 2017 (CISRC '17), 8 pages.

DOI: http://dx.doi.org/10.1145/3064814.3064832

Permission to make digital or hard copies of all or part of this work for personal or classroom use is granted without fee provided that copies are not made or distributed for profit or commercial advantage and that copies bear this notice and the full citation on the first page. Copyrights for components of this work owned by others than ACM must be honored. Abstracting with credit is permitted. To copy otherwise, or republish, to post on servers or to redistribute to lists, requires prior specific permission and/or a fee. Request permissions from permissions@acm.org.

CISRC '17, Oak Ridge, TN, USA

(c) 2017 ACM. 978-1-4503-4855-3/17/04 ..\$15.00

DOI: http://dx.doi.org/10.1145/3064814.3064832

\section{INTRODUCTION}

Software-Defined Networking (SDN) has great potentials to simplify network management based on two distinguished principles. First, it enables programmability of the entire network and second, it separates concerns of the control plane and the data plane [13]. This allows the network to be controlled and configured from a logically centralized unit that can improve policy enforcement and network management. Research in SDN has been increasingly applied to various applications including those in cloud computing and virtualization technologies across multiple backbone networks [19]. While SDN brings benefits and flexibility to network management, the use of a program to define and control network devices also expands security threats [2]. Software is unavoidably vulnerable and has been one of the major causes of many network security breaches. The security of SDN is not only a critical requirement of SDN services but also its architecture.

SDN security has been studied from the perspective of the three SDN architectural layers: Data Plane, Control Plane and Application or Management Plane [13]. Each of these planes and their interfaces are liable to different types of attacks. Much work has focused on security of the controlling unit of the control plane, and the OpenFlow specifying switches for data transmission between the control plane and the data plane $[2,4,5,14,19]$. Some of these security issues are not SDN specific and some are. However, whether they are SDN specific or not, their mitigation techniques in traditional networking and in SDN can differ [8]. See [19] for a broad survey of SDN security issues.

Our research focuses on security in the SDN data plane, which is susceptible to unsolicited attacks that exploit the vulnerability in forwarding devices (switches). By compromising the network switches and intercepting packet transmission, an adversary can eavesdrop undisclosed data at the compromised locations causing data leakages. Even for static eavesdropping where the attacker does not gain access of additional switches, severe information leakages can occur.

Eavesdropping attacks are one of the most common and important network attacks because they are relatively easy to implement and their effects can escalate to more severe attacks such as Denialof-service, side-channel-attacks, and black-holes. There have been relatively few studies on eavesdropping attacks and data leakages in SDN $[4,8,14]$. One focuses on exploiting the overflow vulnerability of the SDN's flow table to derive meta data about packet forwarding [14]. The others address mitigation and detection of leakages during the data transmission in SDN [4, 8]. Although eavesdropping attacks do not occur exclusively in SDN, existing 
solutions in traditional networks are not necessary appropriate for SDN. Similarly, security solutions in SDN may not be applicable or exist in the traditional networking. For example, validating data integrity (to detect malicious acts of eavesdroppers) in the traditional network is very difficult and may not be feasible due to the distributed nature of a large number of switching tables while this can be automatically realized in SDN via its centralized controller [8] Because of high overhead, one approach to address eavesdropping attack is by applying cryptographic network protocols such as $\mathrm{SSH}$. However, such an approach has limitations especially when the network is large and complex [4].

This paper addresses the issue of how to cope with eavesdropping attacks in the SDN data plane by using multiple routing paths (e.g., [4]) to reduce the severity of data leakage. While this existing approach, referred to as a multipath approach, appears to be considerably effective, our simple analysis uncovers that without a proper strategy of data communication, it can still lead to $100 \%$ of data exposure. The paper describes a simple remedy, referred to as a two-way multipath approach, along with illustrations both analytically and experimentally.

The rest of the paper is organized as follows. Section 2 describes the background knowledge of SDN security and an overview of an anti-eavesdropping multipath approach introduced by da Silva et al. [4]. Section 3 introduces a metric for measuring an estimated percentage of data leakage and shows how it can be used to analyze the effectiveness of an anti-eavesdropping approach. This section uncovers security issue of the multipath approach. Section 4 introduces our proposed remedy, a two-way multipath approach. Section 5 presents our experiments to compare the two approaches followed by related work in Section 6 and conclusion in Section 7 .

\section{PRELIMINARY BACKGROUND}

\subsection{SDN: Concepts and Eavesdropping Attacks}

SDN, a relatively new networking fundamental was conceived to meet the challenges of traditional networking to keep up with frequent changes of the network conditions, to perform low-level configuration to comply with high-level network policies, and to efficiently control the network over various tasks [10]. By introducing a concept of programmable networks, SDN enhances the agility in networking management. SDN simplifies complex network configuration and automation by separating concerns of the data plane and the control plane, implementing network switching as packet forwarding, and using centralized software to logically control the entire networks. The centralization of network control and management is one of the key features of SDN development.

As shown in Figure 1, SDN consists of three functional layers: Management Plane, Control Plane and Data (or Forwarding) Plane. The management plane includes applications that allow network developers and operators to define high-level policies of network services such as routing, load balancing and firewalls. These policies are sent to the control plane via the Northbound Advanced Programmable Interfaces (APIs). In the control plane, the controlling unit runs network operating system to provide necessary resources and abstractions to support the controlling functions (e.g., forwarding decisions and load balancing optimization) [13]. The

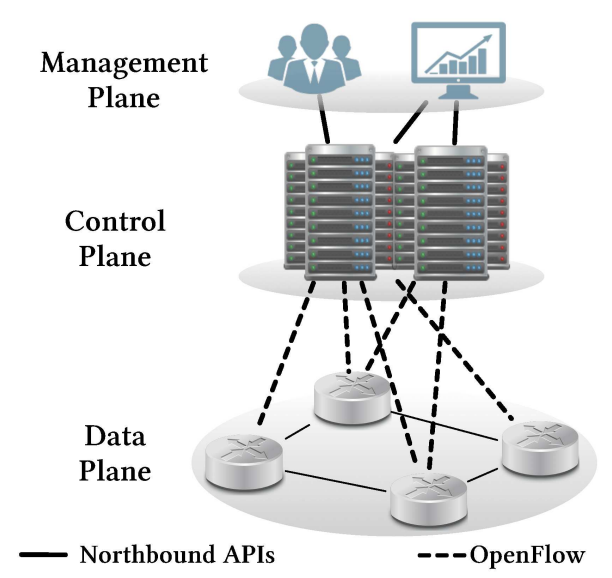

Figure 1: Software-defined networking functional structure

controller adopts OpenFlow protocol $[17,20]$, an open source SDNspecific southbound protocol. It uses OpenFlow to control the traffic and allow direct access to and manipulation of physical and virtual forwarding devices. Communication network is dynamic. Hence, to manage the network effectively, the controller needs to dynamically update the overall structure of the entire network. The controller applies the Link Discovery Service that uses OpenFlow Discovery Protocol to identify the missing links between forwarding devices in the flow path [2].

The data plane includes simple forwarding devices (e.g., switches, routers, middleboxes) and communicates with the control plane via the OpenFlow protocol [11]. For a new flow received via an OpenFlow switch, the routing and forwarding decisions are reactively or proactively instructed by the central controller or distributed controller system $[6,20]$. These instructions are in the form of flow rules in one or more flow tables in the OpenFlow switches in the data plane.

Cyber security of SDN has been studied along different parts of the SDN architecture [19]. Eavesdropping attacks in SDN can occur within the data plane or via communication channels between the controllers in the control plane and forwarding devices in the data plane. In the data plane, eavesdropping attacks can occur in two levels: switches (i.e., forwarding devices) and forwarding links. Once they are compromised and intercepted, the malicious eavesdroppers can listen to data used them for further attacks.

Eavesdropping attacks are common in TCP networks. While cryptography is an accepted mechanism to prevent eavesdropping attacks, methods for eavesdropping detections are not readily realized [8]. Alternatively, many researchers cope with the attacks by improving the network resilience or reducing damage costs (e.g., by moving target strategies [7], or [9]). Similar to traditional networks, anti-eavesdropping counter measures in SDN encompass the same basic stages from prevention (e.g., cryptography at the forwarding device level) to detection (e.g., by verifying integrity of the flow tables) [8], and to mitigating severity (e.g., multipath approach [4]).

Methods successfully employed in traditional networks are not necessarily applicable to SDN (e.g. the protection by encryption 
in SDN has limited scope) [8]. Due to the network programmability and centralized controller in SDN, mechanisms that are attainable in SDN infrastructures may not be easily implemented in traditional networks (e.g. the multipath approach that relies on the controlling unit to redirect flow entries for alternate paths). Next section describes the multipath approach [4] that leads to the main contribution of this paper.

\subsection{Anti-eavesdropping Multipath Approach}

The multipath approach [4] addresses eavesdropping attacks on communication links between forwarding devices in the SDN data plane. In particular, as shown in Figure 2, the approach has been applied to an SDN-based smart grid system that forwards data between the master station and substations.

Given a data transmission from a sender $S$ to a receiver $R$. Typically, the most desirable route is the shortest path between $S$ and $R$. This can be obtained by applying a minimum spanning tree algorithm [6] as indicated by dotted links in Figure 2. Instead of using these paths, the multipath approach employs a timing mechanism to dynamically alternate next shortest path for the transmissions between $S$ and $R$, should there remain data to be transmitted. This mechanism helps prevent a severe case when an eavesdropper is by any chance listening to the data on the shortest route causing all data leakage to the eavesdropper.

We will now illustrate a simple data forwarding using the multipath strategy. Consider a partial SDN Smart Grid network as shown in Figure 2. There are five links connected to Switch 1, which is the only gateway communication to the master station. Suppose $A, B$, $C, D$, and $E$ are listening to packets sent from the master station to substations at the locations indicated in the figure. Based on the spanning tree (i.e., a connected tree whose nodes cover all nodes in the network) of the network, communication paths between each substation and the master station are connected by bold dashed links. Note that there is exactly one path from the master station to each substation. For example, a routing from the master station to substation 10 can only go through switches 1,6 , and 10 but not through switches 1, 3, and 5. Thus, an eavesdropper on any link of the path to a substation can intercept all packets sent to that substation. For instance, eavesdropper $D$ can get all packets in the communication between the master station and substations 2 and 4. Here the SDN data flow network is extremely vulnerable with all packets communicated to the master station being exposed.

To reduce the severity of the data leakage, the multipath approach employs $n$ alternative communication paths between the sender (master station) and the receiver (substation) so that only a portion of the data transmitted is exposed to the eavesdropper. By applying Dijsktra algorithm [4, 6] repeatedly to find the shortest path (and the next shortest path and so on) between the sender and the receiver, the multipath approach continues transmitting the data using next shortest alternate paths until all the data transmission is complete. For example, as shown in Figure 2, when $n=2$, the shortest communication path from the master station to substation 2 is the path $<$ Switch1, Switch2 $>$. The alternative next shortest path is the path $<$ Switch1, Switch7, Switch2 $>$. The packets are alternately sent via these two paths using flow rule to specify a suitable path during a specified timeout period. When the timeout

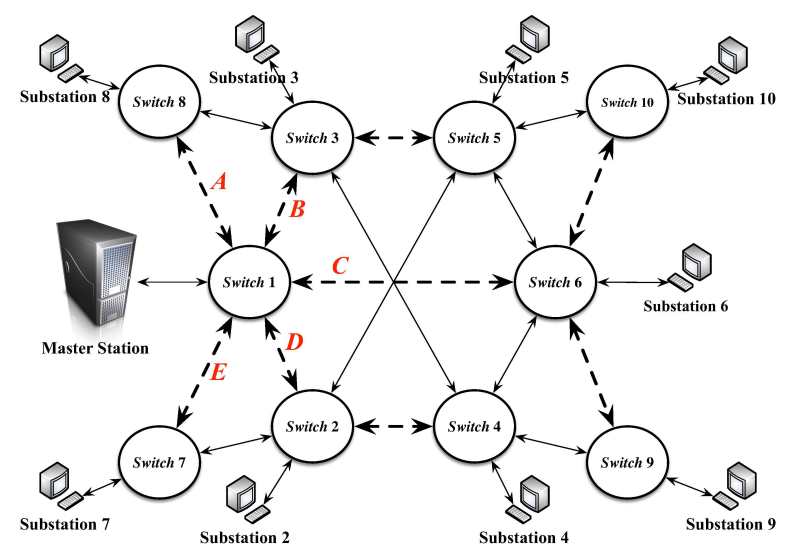

Figure 2: Forwarding devices in smart grid SDN data layer

expires, a new flow rule specifying an alternative path is applied again to continue the data transmission process until all data are received and acknowledged. The number of packets sent through these paths is approximately of equal portions. For example, suppose a total of 100 packets are to be sent via three alternate paths and the data are transmitted at the rate of one packet per second. Given timeout period is five seconds, the number of packets transmitted alternately in the three paths will be 35,35 , and 30 , respectively. In general, when the timeout period is relatively short compared to the time required to send overall number of packets, the number of the packets sent via each alternate route would be reduced approximately by a factor of $\frac{1}{n}$.

\section{THE MULTIPATH ISSUE}

This section describes a simple metric for analyzing data leakage followed by our discovery of an issue of the multipath approach.

\subsection{Simple Analytical Analysis}

Consider a simple analysis of data forwarding in Figure 2 where 100 packets are to be sent from the master station to substation 2 . In this case, as shown in Figure 2, there are two routing paths. The shortest path is $<$ Switch1, Switch2 $>$ of length one and the next shortest path is $<$ Switch1, Switch7, Switch2> of length two. The multipath approach [4] assumes that the acknowledgment packets (ACKs) from the receiver are sent through the shortest path employed (i.e., <Switch1, Switch2> in this case). Thus, when packets sent to substation 2 through the two alternate paths, substation 2 acknowledges the reception of every packet through the same path $<$ Switch1, Switch2>. The number of E's eavesdropped packets is approximately 100/2 (for send) and 0 (for ACKs) for a total of 200 packets sent and acknowledged. This gives $25 \%$ of packets exposed to $E$ and $75 \%$ to $D$, similarly. This is a reduction compared to $100 \%$ exposure in a traditional approach.

We can formulate a metric to estimate the percentage of data exposure as follows. Let $m$ be a total number of data packets to be transmitted from a sender $S$ to a receiver $R$ in the TCP data communication in the data plane. Suppose there are $n$ selected paths for transmitting the data from $S$ to $R$ and $S 1$ and $S 2$ be switches 
(forwarding devices) in the data plane. The estimated percentage of data exposure $E$ of a link $<S 1, S 2>$ is defined as:

$$
E(<S 1, S 2>)=\frac{\left(\frac{m}{n}\right)+k}{2 m}
$$

where $k=0$ if $\langle S 1, S 2>$ is not on the shortest path between $S$ and $R$ (recall that ACKs are returned via shortest path only), otherwise $k=m$. Basically, Equation (1) represents a ratio of the data passing through the link over the total number of data to be transmitted and acknowledged. Based on the estimated data distribution when sending and the multipath assumption when acknowledging the data received, $\frac{m}{n}$ in the top first part of the equation accounts for the data leakage from sending the data from $S$ to $R$ through the link whereas the second part $k$ accounts for the chance of the data leakage caused by ACKs. This simple metric allows an explicit analysis that helps us identify the flaw of the multipath approach. Section 4.2 illustrates the use of this metric through an example.

\subsection{Security Flaw}

Continuing on the scenario introduced in Section 3.1, at first glance the above scenario appears to work perfectly. However, taking a closer look at the ACK packets, the approach of multipath communication can reduce the number of eavesdropped packets only if a link in the (first) shortest path is not compromised. Since all acknowledgment packets are sent through the first shortest path, the eavesdropper can block the ACK packet corresponded to the packet that he did not get, i.e., the packet that sent through alternative path. This will impose the master station (i.e., the sender) to retransmit the packets again via any path, including the shortest path. The eavesdropper only needs to keep blocking ACKs packets until the desired packet is sent through the shortest path. Thus, the eavesdropper will eventually get $100 \%$ of packets even with the implementation of multipath approach. This flaw can cause severe circumstances especially if those captured packets contain sensitive information. For instance, eavesdropper $D$ in Figure 2 can block ACK for the packet sent through the second shortest path and then wait for the master station to retransmit the packet via the link that he is listening to. In addition, $D$ can learn from the intercepted acknowledgments information about the packets transmitted via other paths (e.g., the packet sequence number or window size).

While this flaw is minor and probably occurs by an oversight, recognizing such flaw is not always obvious. Furthermore, when such a flaw can lead to a $100 \%$ of data leakage in certain scenario. This violates the purpose of the anti-eavesdropping approach in SDN network. Next section suggests a simple remedy.

\section{TWO-WAY MULTIPATH APPROACH}

\subsection{The Proposed Algorithm}

To prevent the catastrophic situation presented in section 3.2, we propose a two-way multipath that aims to balance the distribution of packets acknowledged. The SDN's controller will apply the multipath mechanism both ways, i.e., from the sender side and the receiver side. Specifically, data packets and acknowledgements will be sent back and forth via the same transmission path as opposed to using only the first shortest path for ACK packets. Consequently, we will disable the attacker from blocking ACKs addressed in section 3.2 and the percentage of packet exposure will be reduced as well. The proposed solution relies on SDN controller to control the data transmission between the sender and receiver by instructing the receiver side to send the acknowledgment packets via the path used by the sender. It leverages the hard timeout and idle timeout in the flow rules to control path switching (in order of the path length). Consequently, we will disable the attacker from blocking ACKs addressed in section 3.2 and the percentage of packet exposure will be reduced as well. Next we describe the two-way multipath algorithm in more details.

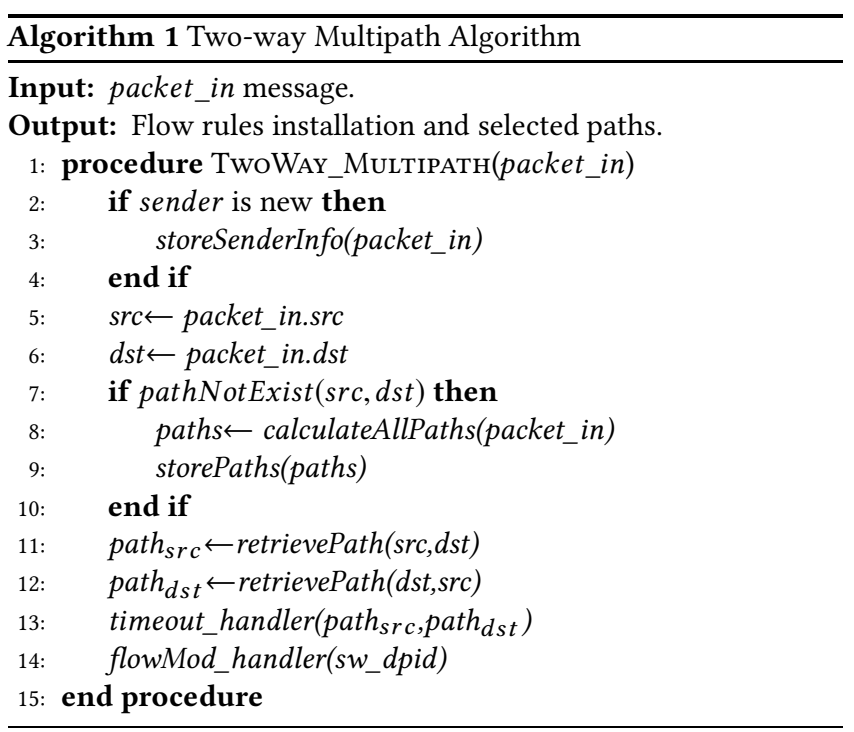

As shown in Algorithm 1, the algorithm takes packet_in message as an input. It first checks against the local dictionary if sender information already exists (line 2). If it does not, it stores the sender information (e.g., MAC address, IP address, and the port number of the switch that is connected to) (line 3). The controller extracts the source (src) and destination (dst) IP addresses from packet_in message (line 5-6) and checks if the paths from source to destination have already been calculated (line 7). If this is not the case, the controller calls calculateAllPaths (line 8) to compute all possible paths between the source and destination in increasing order of path length. Similar to [4], our procedure uses Dijsktra algorithm to generate all shortest paths as follows. The controller runs the algorithm to find the shortest path from the source to destination (the path for acknowledging the receipt of the packets simply stores the path links in reverse order). The path will be marked (e.g., by setting its associated length to a very high value) so that it will not be considered again in the next round. The controller runs Dijsktra algorithm repeatedly until $\mathrm{n}$ possible paths are generated and stored in the controller's repository (line 9). If the paths have already been generated and used in the previous round of data transmission, the algorithm simply retrieves them from its local repository (line 11-12).

Here path src $_{\text {denotes the path from the source while path }}$ sst denotes the paths from the destination (i.e., the reverse of path $\mathrm{p}_{s r c}$ ). 


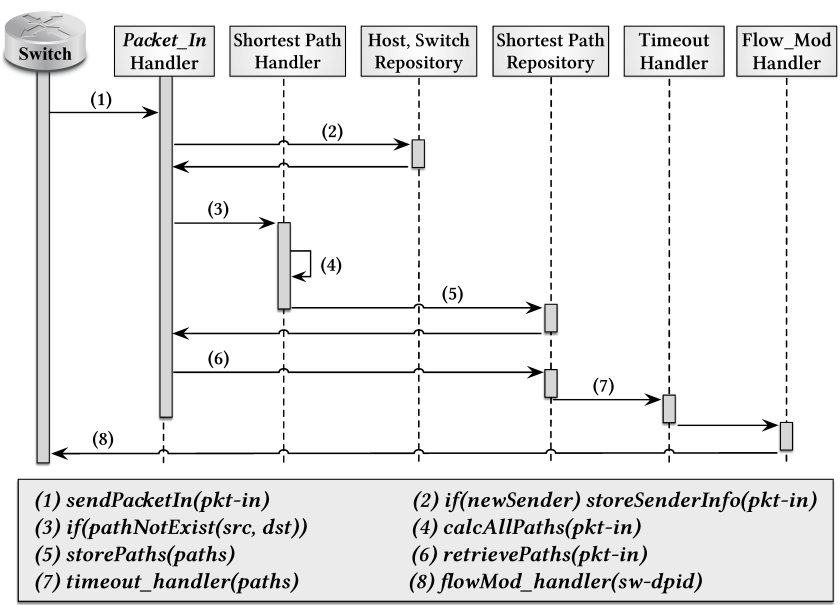

Figure 3: The process of the two-way multipath algorithm

The method timeout_handler $\left(\right.$ path $_{s r c}$, path $\left._{d s t}\right)$ generates the corresponding necessary flow rules (line 13). For the flow rule installation, we adopt two types of flow rules introduced by [4], namely static and dynamic rules. Finally, flowMod_handler is called to install the flow rules to the corresponding switches (line 14).

Figure 3 summarizes activities involved in the two-way multipath approach. The controller continuously maintains an updated network topology including the connectivity among forwarding devices. It also stores information about the senders and receivers in its local repository. When the first packet of a flow is received by a switch, a packet_in message is sent to the controller and handled by packet_in handler. The controller extracts the sender's information (e.g., MAC address, IP address, and port number) and stores them in the local repository. Next, packet_in handler checks if the paths between the sender and receiver are already calculated. If not, shortest path handler calculates the top $n$ shortest paths using Dijkstra algorithm and stores them paths in the local repository. Otherwise, it simply retrieves suitable paths and delivers them to the timeout handler which generates and installs appropriate flow rules accordingly.

The idea of using multiple data transmission routes has been applied to wired and wireless networks to enhance the reliability of the network performance $[15,18]$ (e.g., when dealing with traffic congestion or faulty nodes). However, this paper applies alternative routes to alleviate damages from eavesdropping attacks for different purpose and network infrastructure (i.e., SDN).

\subsection{Illustration and Analytical Results}

Consider a simple example of a transmission from a host (sender) to a server (receiver) in the SDN data plane as shown in Figure 4. Switch 5 is a gateway to the host while Switch 1 is to the server. There are three possible paths of different length between them. Let the selected number of alternative paths $n$ be two. The controller dynamically installs and updates flow rules at each switch on the path to direct data transmission using first the shortest path $<$ Switch 5, Switch $1>$. Once the packets are received at the server, ACKs will be sent in the reversed route back to the sender (host).

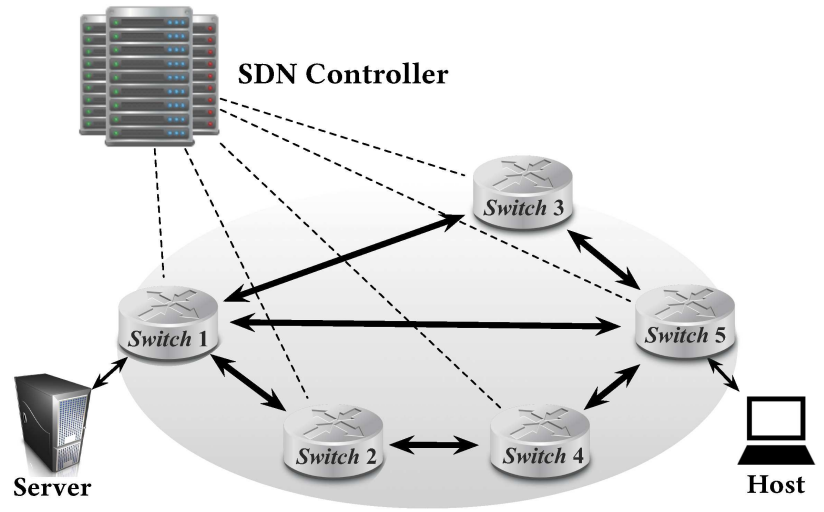

Figure 4: Three-forwarding-route example

When timeout occurs, the controller updates new flow rules to switch to an alternative path $<$ Switch 5 , Switch 3 , Switch $1>$, which is the next shortest path. The process keeps alternating between these two paths until all data transmission is complete.

We provide a simple analysis on this example to compare the effectiveness of the multipath and the two-way multipath approaches. Suppose a total of 100 packets is to be sent from the host to the server. Let $S 1$ denote Switch 1 and this similarly applies to other switches. For simplicity, we assume there is no communication traffic congestion and that the timeout period is very short compared to the overall time required to send all packets (i.e., fast transmission rate). Using the metric formulated in Section 3.1, we can compute the percentages of data exposure. For the multipath approach, $E(<S 5, S 1>)=(100 / 2+100) / 200=75 \%$ and $E(<S 5, S 3>)=$ $(100 / 2+0) / 200=25 \%$. Since the paths and links are disjoint, we can conclude that the data exposure of the shortest path $\langle S 5, S 1>$ and the alternate path $<S 5, S 3, S 1>$ is $75 \%$ and $25 \%$, respectively. For the two-way multipath approach, $E(<S 5, S 1>)=(100 / 2+100 / 2) / 200=$ $50 \%$ and $E(<S 5, S 3>)=(100 / 2+100 / 2) / 200=50 \%$. By similar argument, the data exposure of the shortest path $\langle S 5$, S1 $>$ and the alternate path $<S 5, S 3, S 1>$ is each $50 \%$. This agrees with the design strategy to make the number of packets through each link in a more balanced distribution in order to reduce the risk of damage if the adversary intercepts a critical path.

\section{EXPERIMENTS}

\subsection{Experimental Setup}

The experiment is implemented using Mininet [21] for emulating virtual SDN/OpenFlow networks. The forwarding devices are Open vSwitch [22] and the controller is POX [16], a Python-based controller dedicated for SDN. The speed of the communication links was set to $1 \mathrm{Gbps}$. We assume the links have enough bandwidth and the network has no traffic congestion. The experiment was run on Ubuntu VM 14.04 on MacBook 2.2GHz i7 with 16GB of memory.

In our experiment scenario, data packets are transmitted from a host to a web server. Particularly, the host sends HTTP GET requests, using Unix command wget, to the web server every $15 \mathrm{sec}-$ onds. The server responds by sending a page of size (398 byte). For comparison purposes, we ran three different types of experiments 


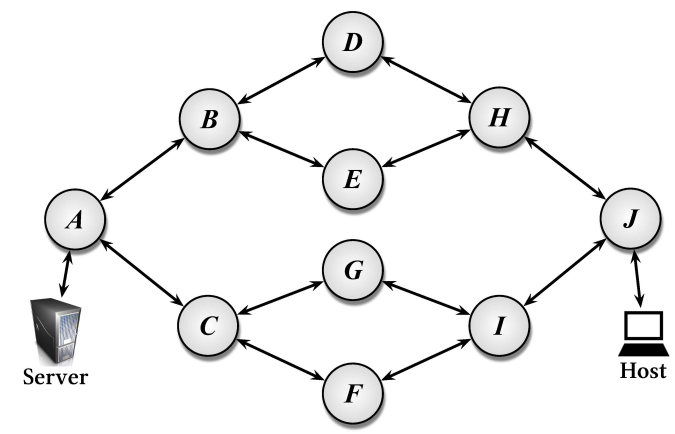

Figure 5: The network topology used in the simulation

using three approaches: (1) traditional shortest path only, (2) the multipath approach and (3) the proposed two-way multipath approach. As shown in Figure 5, our network topology consists of 10 OpenFlow-based switches (A to J) and the total possible transmission paths between the host to the server are four. In the traditional approach, we simulate the controller that applies spanning tree algorithm for unicast routing between the server and the host. For each of the multipath approaches, we ran experiments with the number of alternate paths $n$ from two to four with idle and hard timeouts of 5 and 10 seconds, respectively. The total time for running each experiment was set for 10 minutes. Note that the selected timeout periods are short compared to the time required to send overall number of packets we set in our experiments. Thus, we expect to see a pretty well balanced distribution of the number of packets at the switch with splitting paths.

\subsection{Experimental Results}

Results of our simulations are divided into two aspects: (1) evaluation of the effectiveness of the proposed approach in alleviating effects from eavesdropping attacks, and (2) performance in terms of overheads and effects on SDN operations in the data plane. For the first aspect, Table 1 compares results obtained from all the three approaches (i.e., the single shortest path using spanning tree algorithm, the multipath approach, and the proposed two-way multipath approach) for different number of alternate paths from $n=2$ to 4 . Each entry in the table represents the number of packets transmitted through the corresponding link (e.g., link $\langle A, B\rangle$ is denoted by $A B$ ) and its percentage of data exposure shown in parentheses.

As shown in Figure 5, there are four paths between the host and the server and each of them has equal length (although our approach should be able to handle alternate paths of unequal length effectively). For convenience, our implementation selects the top $n$ paths as shown in Figure 5 to be alternate paths. For example, for $n=3$, the top three paths considered for transmission are $A B D H \mathcal{F}$, $A B E H f$, and $A C G I f$ (If data transmission is not completed, the next route will start again from the first path $\mathrm{ABDHJ}$ and so on). This is the reason why some links are not applicable (na) in Table 1. For example, for $n=3$, the most bottom path of Figure 5, namely $A C F I f$ is omitted. This leaves links $C F$ and FI unused (not applicable) as reflected in Table 1.

As shown in Table 1, unlike the single route method, both of the multipath approaches reduce the percentages of leakages to be less
Table 1: Comparing data exposures in all approaches

\begin{tabular}{|c|c|c|c|c|c|c|c|}
\hline \multirow[b]{2}{*}{ Links } & \multirow[b]{2}{*}{$\begin{array}{l}\text { Single } \\
\text { Route }\end{array}$} & \multicolumn{2}{|c|}{$n=2$} & \multicolumn{2}{|c|}{$n=3$} & \multicolumn{2}{|c|}{$n=4$} \\
\hline & & $\begin{array}{l}\text { Multi- } \\
\text { path }\end{array}$ & $\begin{array}{l}\text { Two-way } \\
\text { Multipath }\end{array}$ & $\begin{array}{l}\text { Multi- } \\
\text { path }\end{array}$ & $\begin{array}{l}\text { Two-way } \\
\text { Multipath }\end{array}$ & $\begin{array}{l}\text { Multi- } \\
\text { path }\end{array}$ & $\begin{array}{l}\text { Two-way } \\
\text { Multipath }\end{array}$ \\
\hline$A B$ & $\begin{array}{c}754 \\
(100 \%)\end{array}$ & $\begin{array}{c}600 \\
(75.9 \%)\end{array}$ & $\begin{array}{c}339 \\
(47.5 \%)\end{array}$ & $\begin{array}{c}732 \\
(83.3 \%)\end{array}$ & $\begin{array}{c}530 \\
(67.4 \%)\end{array}$ & $\begin{array}{c}598 \\
(75.6 \%)\end{array}$ & $\begin{array}{c}392 \\
(51.4 \%)\end{array}$ \\
\hline$B D$ & $\begin{array}{c}754 \\
(100 \%)\end{array}$ & $\begin{array}{c}600 \\
(75.9 \%)\end{array}$ & $\begin{array}{c}339 \\
(47.5 \%)\end{array}$ & $\begin{array}{c}516 \\
(66.9 \%)\end{array}$ & $\begin{array}{c}274 \\
(34.9 \%)\end{array}$ & $\begin{array}{c}502 \\
(63.5 \%)\end{array}$ & $\begin{array}{c}200 \\
(26.2 \%)\end{array}$ \\
\hline$D H$ & $\begin{array}{c}754 \\
(100 \%)\end{array}$ & $\begin{array}{c}600 \\
(75.9 \%) \\
\end{array}$ & $\begin{array}{c}339 \\
(47.5 \%)\end{array}$ & $\begin{array}{c}516 \\
(66.9 \%)\end{array}$ & $\begin{array}{c}274 \\
(34.9 \%)\end{array}$ & $\begin{array}{c}502 \\
(63.5 \%)\end{array}$ & $\begin{array}{c}200 \\
(26.2 \%)\end{array}$ \\
\hline$H J$ & $\begin{array}{c}754 \\
(100 \%)\end{array}$ & $\begin{array}{c}600 \\
(75.9 \%)\end{array}$ & $\begin{array}{c}339 \\
(47.5 \%)\end{array}$ & $\begin{array}{c}732 \\
(83.3 \%)\end{array}$ & $\begin{array}{c}530 \\
(67.4 \%)\end{array}$ & $\begin{array}{c}598 \\
(75.6 \%)\end{array}$ & $\begin{array}{c}392 \\
(51.4 \%)\end{array}$ \\
\hline$B E$ & na & na & na & $\begin{array}{c}126 \\
(16.3 \%)\end{array}$ & $\begin{array}{c}256 \\
(32.6 \%)\end{array}$ & $\begin{array}{c}96 \\
(12.1 \%)\end{array}$ & $\begin{array}{c}192 \\
(25.2 \%)\end{array}$ \\
\hline$E H$ & na & na & na & $\begin{array}{c}126 \\
(16.3 \%)\end{array}$ & $\begin{array}{c}256 \\
(32.6 \%) \\
\end{array}$ & $\begin{array}{c}96 \\
(12.1 \%) \\
\end{array}$ & $\begin{array}{c}192 \\
(25.2 \%) \\
\end{array}$ \\
\hline$A C$ & na & $\begin{array}{c}190 \\
(24.1 \%)\end{array}$ & $\begin{array}{c}375 \\
(52.5 \%)\end{array}$ & $\begin{array}{c}129 \\
(16.7 \%)\end{array}$ & $\begin{array}{c}256 \\
(32.6 \%)\end{array}$ & $\begin{array}{c}193 \\
(24.4 \%)\end{array}$ & $\begin{array}{c}370 \\
(48.5 \%)\end{array}$ \\
\hline$C F$ & na & na & na & na & na & $\begin{array}{c}99 \\
(12.5 \%)\end{array}$ & $\begin{array}{c}178 \\
(23.4 \%)\end{array}$ \\
\hline$F I$ & na & na & na & na & na & $\begin{array}{c}99 \\
(12.5 \%)\end{array}$ & $\begin{array}{c}178 \\
(23.4 \%)\end{array}$ \\
\hline$I J$ & na & $\begin{array}{c}190 \\
(24.1 \%)\end{array}$ & $\begin{array}{c}375 \\
(52.5 \%)\end{array}$ & $\begin{array}{c}129 \\
(16.7 \%)\end{array}$ & $\begin{array}{c}256 \\
(32.6 \%)\end{array}$ & $\begin{array}{c}193 \\
(24.4 \%)\end{array}$ & $\begin{array}{c}370 \\
(48.5 \%)\end{array}$ \\
\hline$C G$ & na & $\begin{array}{c}190 \\
(24.1 \%)\end{array}$ & $\begin{array}{c}375 \\
(52.5 \%)\end{array}$ & $\begin{array}{c}129 \\
(16.7 \%)\end{array}$ & $\begin{array}{c}256 \\
(32.6 \%)\end{array}$ & $\begin{array}{c}94 \\
(11.9 \%)\end{array}$ & $\begin{array}{c}192 \\
(25.2 \%)\end{array}$ \\
\hline$G I$ & & $\begin{array}{c}190 \\
(24.1 \%)\end{array}$ & $\begin{array}{c}375 \\
(52.5 \%)\end{array}$ & $\begin{array}{c}129 \\
(16.7 \%)\end{array}$ & $\begin{array}{c}256 \\
(32.6 \%)\end{array}$ & $\begin{array}{c}94 \\
(11.9 \%)\end{array}$ & $\begin{array}{c}192 \\
(25.2 \%)\end{array}$ \\
\hline
\end{tabular}

than $100 \%$ (ranging from about $12 \%$ to $76 \%$ when all four paths are used). The maximum (minimum) percentages of data exposed by the multipath (two-way multipath) approach decreases from $75.9 \%$ to $83.3 \%$ and to $75.6 \%$ (24.1\% to $16.3 \%$ and to $12.5 \%$ ) as $n$ increases from 2 to 3 and to 4 . Thus, the average percentage of data leakages decreases as $n$ increases, as it should be. The same can be said for the two-way multipath approach but with the smaller average of percentage of data exposure. The main difference between the multipath and the two-way multipath approaches is that the percentages of leakages in the latter approach are overall more evenly distributed according to our strategy to avoid catastrophic blocking. Some links may increase data exposure while some may reduce. To see this more clearly, Figure 6 compares data exposure of the two approaches when $n=2$ and $n=3$.

The Figure 6 top part uses two top paths that are symmetry and completely disjoint. The results show that although the multipath approach reduces the data exposure, the links on the topmost path $A B D H f$ still considerably has higher percentage of data exposure than that of links on the bottom alternate path ACGIf. On the other hand, the percentage of data exposure of the two-way multipath approach is about the same on each link. Thus, the latter is less vulnerable because there is no one link that is highly critical should it be compromised and intercepted.

The bottom part of Figure 6 shows similar results. The links $A B$ and $H f$ are shared for two paths and thus, have the highest traffic compared to other links for both approaches. The data exposures in $A B, H f, B D$ and $D H$ are high in the multipath approach because they are on the only path used for ACKs. However, this is not the case for the two-way multipath approach. 


\section{Comparisons when $n=2$}

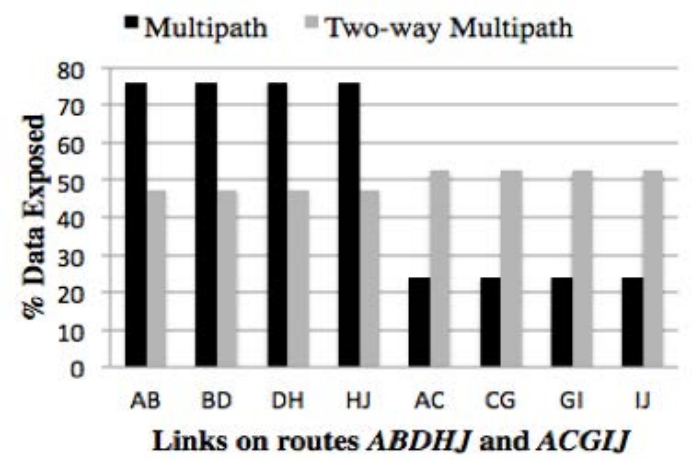

Comparisons when $n=3$

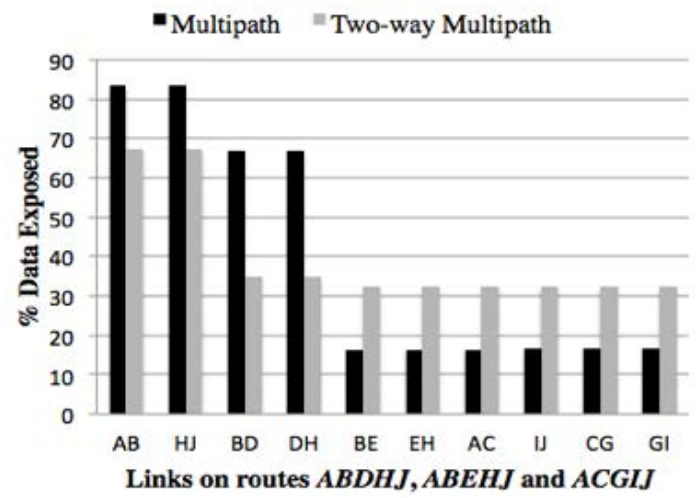

Figure 6: Comparing data exposure using alternate paths

For the second aspect of the experiments to assess performance, overheads and how the two-way multipath approach effects SDN functions, we ran experiments to observe various performance measures as introduced in [4]. For the next experiment we show results obtained when $n=4$ to assess the performance when the proposed multipath approach is highly utilized.

As shown in Figure 7, the number of rules installed at a time fluctuates over time with the same pattern with or without using alternate paths. This is because the number of flow rules installedin and removed-from switches are consistent and almost identical

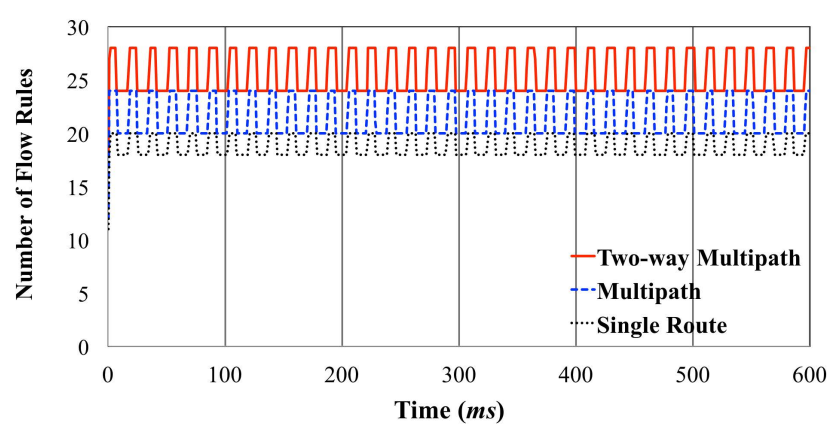

Figure 7: Total number of rules installed at a time at the same given time. However, the number of flow rules for the two-way multipath approach is higher than that of the multipath approach. The number of flow rules with the traditional spanning tree approach is the lowest as expected.

Figure 8 shows that, in our simulation, the number of packet_ins is about the same for the two multipath approaches with the twoway one being slightly higher when $n=2$. The number of packet_ins helps explained the following results on the traffics. As shown in Figure 9, the average traffic in control channels depends on the number of packet_ins and thus, the comparison results of the traffic in control channels are similar to that of the results in Figure 8. As expected, the two-way multipath approach has higher average traffics than those of the multipath approach and as $n$ increases their differences decrease. This is because for large $n$, traffics in both approaches are more similar in that they are more widely and evenly distributed making the difference due to ACKs traffics less apparent.

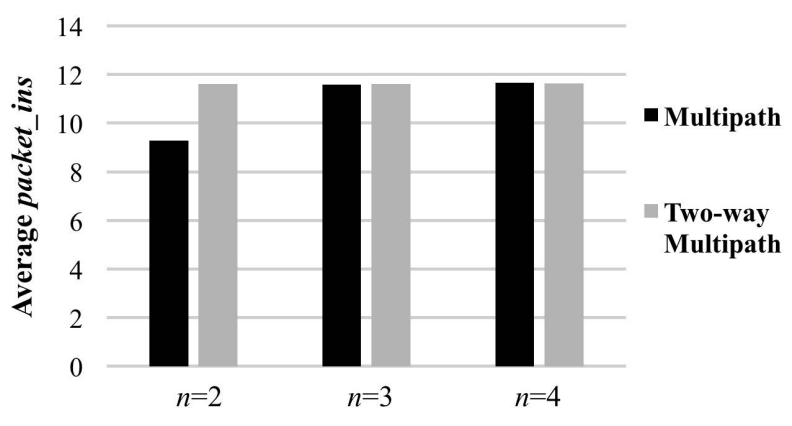

Figure 8: Average number of packet-ins

Figure 10 shows that the percentages of traffic retransmission of the two-way multipath approach are less than those of the multipath approach when $n$ is even, but this is not the case when $n$ is odd. This could be because of the symmetry of the network topology in our experiments. When $n$ is odd, the traffic is not as evenly distributed causing congested communication traffic in the links that are shared by multiple paths. In such a case, the two-way multipath approach would have higher percentages of traffic retransmission as shown in Figure 10. However, the traffic retransmission largely depends on the topology of the network.

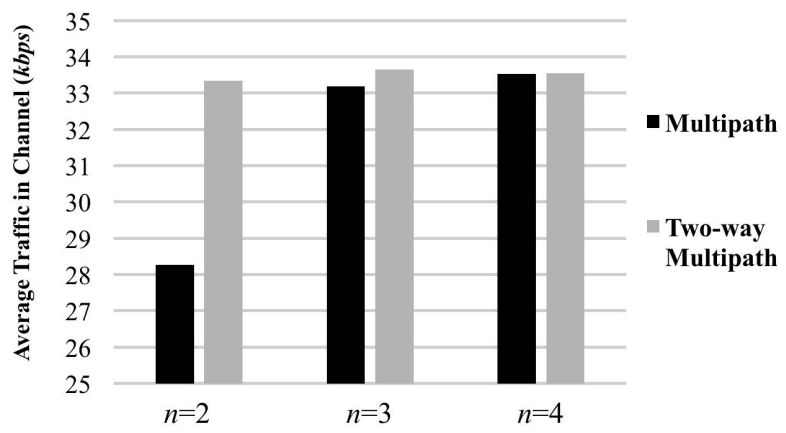

Figure 9: Average traffic in control channels (kbps) 


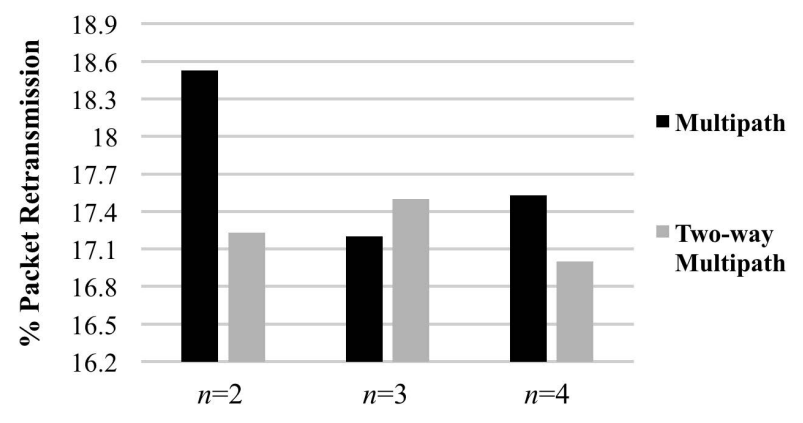

Figure 10: Percentages of traffic retransmission

In general, we see the proposed two-way multipath approach is more effective in terms of reducing high percentages of data exposure in certain paths making SDN less vulnerable and yet it does not appear to impair the performance of SDN forwarding mechanisms.

\section{RELATED WORK}

Much research has addressed security issues in the SDN data plane $[1,2,12,14,19]$. Eavesdropping attacks in the SDN data plane have been studied in the context of data leakages at various stages of the attacks. Prior the attacks, preventative techniques mostly rely on cryptography although their applicability can be limited [8]. During the attacks, Po-Wen et al. [3] introduced a mechanism to detect compromised OpenFlow switches. Posting the attacks, Benton et al. [2] and Kreutz [12] show how eavesdropping through a compromised switch can lead to flow table modification that results in other more severe attacks such as Denial-of-Service. Leng, et al. [14] show mechanisms to infer the flow table capacity and usage. Many researchers cope with the attacks by improving the network resilience, e.g., by moving target strategies $[7,9]$ or employing multiple paths $[4,15]$. Work by da Silva et al. [4] is the closest to our proposed approach in that both use alternative multiple paths.

\section{CONCLUSION}

The ability of SDN to ease network control and management has gained a considerable momentum from of the networking community. On the other hand, security issues remain challenging. We have pointed out a security flaw in the multipath approach [4] that can lead to $100 \%$ of information leakage. While this could be a minor oversight, discovering it is not always obvious. We have also proposed a metric that can be used for analyzing the estimated degrees of leakages in the SDN forwarding plane. We have proposed two-way multipath approach to fix the security issue and evaluate the approach analytically and empirically. The results show that our two-way multipath approach can avoid the case of $100 \%$ captured data as well as reducing the percentages of data exposure compared with the multipath approach. In future work, we shall include more complex and realistic networks.

\section{ACKNOWLEDGMENT}

The first author is thankful for the support from his $2^{\text {nd }}$ affiliation, Prince Sattam bin Abdulaziz University. The second author would like to thank the Thailand Research Fund and King Mongkut's University of Technology Thonburi for their support through the Royal Golden Jubilee Ph.D. Program (grant\# PHD/0031/2553).

\section{REFERENCES}

[1] Markku Antikainen, Tuomas Aura, and Mikko Särelä. 2014. Spook in your network: Attacking an sdn with a compromised openflow switch. In Nordic Conference on Secure IT Systems. Springer, 229-244.

[2] Kevin Benton, L Jean Camp, and Chris Small. 2013. Openflow vulnerability assessment. In Proceedings of the second ACM SIGCOMM workshop on Hot topics in software defined networking. ACM, 151-152.

[3] Po-Wen Chi, Chien-Ting Kuo, Jing-Wei Guo, and Chin-Laung Lei. 2015. How to detect a compromised SDN switch. In Network Softwarization (NetSoft), 2015 1st IEEE Conference on. IEEE, 1-6.

[4] Eduardo Germano da Silva, Luis Augusto Dias Knob, Juliano Araujo Wickboldt, Luciano Paschoal Gaspary, Lisandro Zambenedetti Granville, and Alberto Schaeffer-Filho. 2015. Capitalizing on SDN-based SCADA systems: An anti-eavesdropping case-study. In Integrated Network Management (IM), 2015 IFIP/IEEE International Symposium on. IEEE, 165-173.

[5] Mohan Dhawan, Rishabh Poddar, Kshiteej Mahajan, and Vijay Mann. 2015. SPHINX: Detecting Security Attacks in Software-Defined Networks.. In NDSS.

[6] Nick Feamster, Jennifer Rexford, and Ellen Zegura. 2014. The road to SDN: an intellectual history of programmable networks. ACM SIGCOMM Computer Communication Review 44, 2 (2014), 87-98.

[7] Jafar Haadi Jafarian, Ehab Al-Shaer, and Qi Duan. 2012. Openflow random host mutation: transparent moving target defense using software defined networking. In Proceedings of the first workshop on Hot topics in software defined networks. ACM, 127-132.

[8] Fan Jiang, Chen Song, Hao Xun, and Zhen Xu. 2016. Combat-Sniff: A Comprehensive Countermeasure to Resist Data Plane Eavesdropping in SoftwareDefined Networks. American fournal of Networks and Communications 2, 5 (2016), 27-34.

[9] Panos Kampanakis, Harry Perros, and Tsegereda Beyene. 2014. SDN-based solutions for moving target defense network protection. In A World of Wireless, Mobile and Multimedia Networks (WoWMoM), 2014 IEEE 15th International Symposium on. IEEE, 1-6.

[10] Hyojoon Kim and Nick Feamster. 2013. Improving network management with software defined networking. IEEE Communications Magazine 51, 2 (2013), 114119.

[11] Teemu Koponen, Martin Casado, Natasha Gude, Jeremy Stribling, Leon Poutievski, Min Zhu, Rajiv Ramanathan, Yuichiro Iwata, Hiroaki Inoue, Takayuki Hama, and others. 2010. Onix: A distributed control platform for largescale production networks.. In OSDI, Vol. 10. 1-6.

[12] Diego Kreutz, Fernando Ramos, and Paulo Verissimo. 2013. Towards secure and dependable software-defined networks. In Proceedings of the second ACM SIGCOMM workshop on Hot topics in software defined networking. ACM, 55-60.

[13] Diego Kreutz, Fernando MV Ramos, Paulo Esteves Verissimo, Christian Esteve Rothenberg, Siamak Azodolmolky, and Steve Uhlig. 2015. Software-defined networking: A comprehensive survey. Proc. IEEE 103, 1 (2015), 14-76.

[14] Junyuan Leng, Yadong Zhou, Junjie Zhang, and Chengchen Hu. 2015. An inference attack model for flow table capacity and usage: Exploiting the vulnerability of flow table overflow in software-defined network. arXiv preprint arXiv:1504.03095 (2015).

[15] Wenjing Lou and Yuguang Fang. 2001. A multipath routing approach for secure data delivery. In Military Communications Conference, 2001. MILCOM 2001. Communications for Network-Centric Operations: Creating the Information Force. IEEE, Vol. 2. IEEE, 1467-1473.

[16] James McCauley and Apache License V2.0. 2012. POX Controller. (2012).

[17] Nick McKeown, Tom Anderson, Hari Balakrishnan, Guru Parulkar, Larry Peterson, Jennifer Rexford, Scott Shenker, and Jonathan Turner. 2008. OpenFlow: enabling innovation in campus networks. ACM SIGCOMM Computer Communication Review 38, 2 (2008), 69-74.

[18] Peter P Pham and Sylvie Perreau. 2003. Performance analysis of reactive shortest path and multipath routing mechanism with load balance. In INFOCOM 2003. Twenty-Second Annual foint Conference of the IEEE Computer and Communications. IEEE Societies, Vol. 1. IEEE, 251-259.

[19] Sandra Scott-Hayward, Gemma O'Callaghan, and Sakir Sezer. 2013. SDN security: A survey. In Future Networks and Services (SDN4FNS), 2013 IEEE SDN For. IEEE, 1-7.

[20] OpenFlow Switch Specification. 2014. 1.5.1 (Protocol version 0x06), December, 2014. (2014).

[21] Mininet Team. 2014. Mininet Emulator. (2014)

[22] OPEN VSWITCH. 2013. Open vSwitch. (2013). 